\title{
Clinical Spectrum and Outcomes of Ocular and Periocular Complications following External- Beam Radiotherapy for Inoperable Malignant Maxillary Sinus Tumors
}

\author{
Darren Shu Jeng Ting a, b Romeela Rana-Rahman ${ }^{b}$ Jia Yu Ng ${ }^{b}$ \\ David J.P. Wilkinson ${ }^{c}$ Desiree Ah-Kine ${ }^{b}$ Trushar Patel $^{\mathrm{b}}$ \\ ${ }^{a}$ Academic Ophthalmology, Division of Clinical Neuroscience, School of Medicine, University of Nottingham, \\ Nottingham, UK; ${ }^{b}$ Department of Ophthalmology, James Cook University Hospital, Middlesbrough, UK; \\ 'Department of Radiotherapy and Oncology, James Cook University Hospital, Middlesbrough, UK
}

\section{Keywords \\ Amniotic membrane - Limbal stem cell deficiency · \\ Maxillary tumor · Neurotrophic keratopathy · Paranasal sinus tumor · Radiation keratopathy · Radiotherapy}

\begin{abstract}
Purpose: To highlight the clinical spectrum, management, and outcomes of ocular/periocular complications following high-dose external-beam radiotherapy (EBRT) for inoperable malignant maxillary sinus-involving tumors (MMST). Methods: A retrospective, interventional case series. All patients who were diagnosed with inoperable MMST (with orbital involvement) and treated with high-dose fractionated EBRT (65 Gy in 30 fractions) at James Cook University Hospital, UK, were included. Results: Seven patients with advanced MMST (T4aNOM0-T4bN2cM0) were included and were followed up for $23.8 \pm 10.2$ months. Severe lid margin disease, dry eye, and neurotrophic keratopathy were universally observed. Other complications included cicatricial conjunctivitis (71\%), corneal perforation (57\%), limbal stem cell deficiency (LSCD; 43\%), glaucoma (29\%), and superimposed candida keratitis (14\%). Amniotic membrane transplant (AMT; 71\%), tarsorrhaphy (43\%), tectonic keratoplasty (29\%),
\end{abstract}

and evisceration (14\%) were warranted. Intact corneal epithelium was observed in all patients and good correcteddistance visual acuity ( $\geq 20 / 60)$ was observed in $3(43 \%)$ patients at final follow-up. Conclusion: High-dose EBRT for inoperable MMST can lead to a wide array of severe ocular/ periocular complications. AMT serves as a potentially useful treatment modality to restore the ocular surface integrity after severe radiation keratopathy. We advocate active monitoring for any evolving ophthalmic complications during and after EBRT to enable timely intervention.

(c) 2020 S. Karger AG, Basel

\section{Introduction}

Paranasal sinus tumor is an uncommon type of head and neck cancer, with an estimated incidence rate of 2 per million people per year [1]. It represents $3 \%$ of all malignancies of the head and neck. The majority are squamous cell carcinomas (SCCs) affecting the maxillary sinuses, but a wide variety of histological tumor types can occur in the sinonasal tract [2]. As paranasal sinus tumors often manifest at a late stage, the orbit is commonly affected due to the proximity of these structures [3]. According to

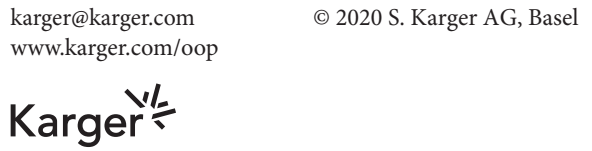


the UK national guideline, surgical resection with or without orbital exenteration followed by adjuvant radiotherapy (with concurrent platinum chemotherapy in selected patients with SCC) serves as the mainstay of treatment for paranasal sinus tumor [2]. However, in inoperable cases where the risks substantially outweigh the benefit of the surgery, or if the patient refuses surgery, primary radiotherapy with or without chemotherapy can be used for local control and occasionally achieves cure [4].

External-beam radiotherapy (EBRT) is a treatment modality that uses ionizing radiation (most commonly high-energy X-ray beams, known as photons) to treat diseases, including cancer. The utility of ionizing radiation in cancer treatment is based on the rationale that cancer cells proliferate rapidly, rendering them more sensitive to radiation-induced DNA damage than normal tissues. Radiotherapy mainly acts by damaging the cancer cells and halting their divisional ability via direct damage to the DNA or indirect cellular damage by the production of free radicals [5]. The ultimate goal of radiotherapy is to destroy cancer cells while minimizing collateral damage to the surrounding tissues [6]. With the technological developments in EBRT, including the conformity to a treatment volume (known as intensitymodulated radiotherapy [IMRT]), radiation dose can now be shaped more conformally to a target volume, improving the therapeutic ratio $(\mathrm{TR})$ of radiotherapy $(\mathrm{TR}=$ probability of tumor control/probability of host tissue damage) $[7,8]$.

Radiotherapy is widely used in the field of ophthalmology for thyroid eye disease, choroidal melanoma, retinoblastoma, lacrimal gland carcinoma, and orbital tumors, amongst others $[9,10]$. The ophthalmic and adnexal complications of radiotherapy for various benign and malignant ocular and periocular diseases have been described in the literature [9-11]; however, there are only limited reports on the ophthalmic complications following radiotherapy to the maxillary sinus region [12]. With improvements in radiotherapy technology, patients with advanced inoperable maxillary sinus-involving tumors (MMST) are now more likely to survive with preservation of the eye. However, that means that ophthalmologists are now increasingly faced with the ocular and periocular complications of high-dose radiotherapy for these tumors. In view of the scarcity of information in the literature, we aimed to highlight the clinical spectrum, management, and outcomes of ocular and periocular complications following EBRT for inoperable MMST with orbital involvement.

Ocular Complications of Radiotherapy for Malignant MST

\section{Materials and Methods}

This was a retrospective interventional case series. All consecutive patients diagnosed with inoperable MMST and treated with high-dose fractionated EBRT, and undergoing IMRT and/or chemotherapy at James Cook University Hospital, UK, between August 2015 and February 2018, were included. Relevant data, including the demographic factors, dosage, and regime of radiotherapy and/or chemotherapy, stage of tumor, histopathologic diagnosis, ocular and periocular complications, management, and ocular and systemic treatment outcomes, were collected and analyzed. Tumors were graded according to the American Joint Committee on Cancer/International Union against Cancer (AJCC/ UICC) 8th Edition manual [13].

\section{Ophthalmic Assessment}

Slit-lamp photography was performed for assessment and monitoring purposes. Central corneal sensation of the studied eyes was measured using either a Cochet-Bonnet esthesiometer (CBE; Western Ophthalmics, Washington, DC, USA) or a cotton wool wisp, and was compared to the fellow unaffected eye as a reference. Neurotrophic keratopathy was graded as I, II, or III according to Mackie's classification [14]. Limbal stem cell deficiency (LSCD) was assessed and diagnosed based on a combination of clinical signs and/or immunohistochemical analysis, particularly examining for increased expression of conjunctival epithelial cell markers (cytokeratin [CK]-13 and CK-19), decreased expression/the absence of corneal epithelial cell markers (CK-3 and CK-12), and the presence of conjunctival goblet cells [15]. Intact corneal epithelium was defined as the absence of frank corneal epithelial defect.

\section{Results}

\section{Patients' Demographic Factors and Details of Cancer Treatment}

A total of 7 patients were included in this study; the mean age of the patients was $67.8 \pm 12.8$ years (range $49.7-82.2$ years) and $5(71 \%)$ were male (Table 1$)$. The mean follow-up duration was $23.8 \pm 10.2$ months (range 10.1-33.0 months). All patients had a histologically confirmed diagnosis of advanced MMST (T4aN0M0T4bN2cM0) with orbital involvement, predominantly caused by SCC $(6,86 \%)$. Five $(71 \%)$ were cases of primary maxillary sinus tumor; 2 (29\%) cases involved the maxillary sinuses, extending either from the nasal cavity $(\mathrm{Pa}-$ tient 2) or with involvement of all paranasal sinuses, the nasal cavity, nasopharynx, and the base of the skull (Patient 1). All patients underwent high-dose fractionated EBRT, using IMRT ( 65 Gy in 30 fractions over 6 weeks), which was the standard dose prescription for treating inoperable paranasal sinus tumors [2], and $5(71 \%)$ patients received concurrent cisplatin $\left(35 \mathrm{mg} / \mathrm{m}^{2}\right)$ chemotherapy. An example of the treatment plan using IMRT is shown in Figure 1.

Ocul Oncol Pathol 2021;7:36-43

DOI: $10.1159 / 000511011$ 
Fig. 1. Transverse view of a treatment plan for Patient 2 delivering 65 Gy in 30 fractions to the planning target volume (PTV) using intensity-modulated radiotherapy (IMRT). Gross tumor volume (GTV), green; clinical target volume (CTV), yellow; PTV, red. In this case, the GTV extends superiorly and can be seen involving the ethmoid sinuses and nasal cavity medial to the globe. Isodose lines are demonstrated as a color wash according to the key on the left of the image. The globes and left lens are also outlined.

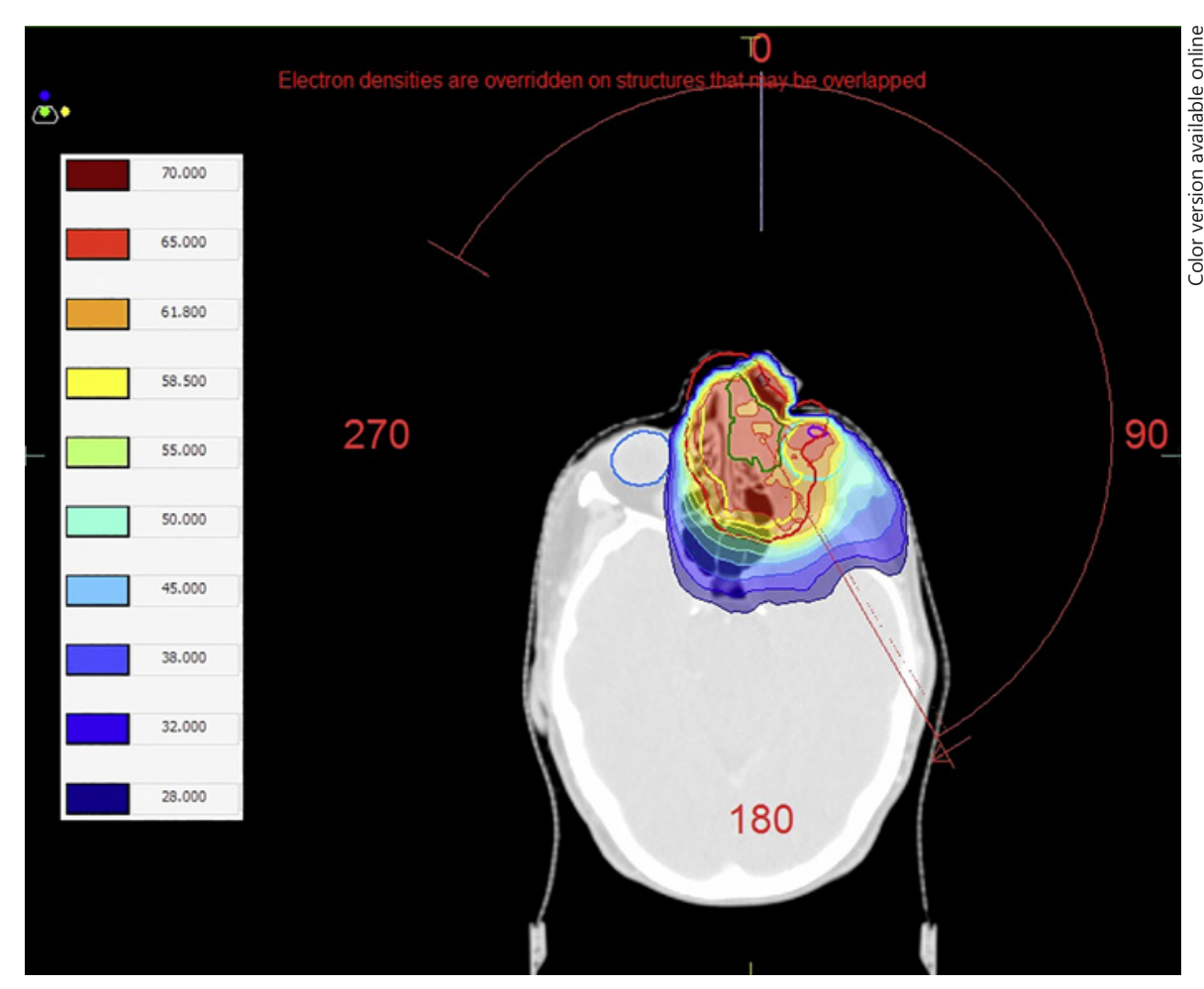

Table 1. A summary of the clinical findings and outcomes of patients who underwent high-dose radiotherapy for inoperable malignant maxillary sinus-involving tumor

\begin{tabular}{|c|c|c|c|c|c|c|c|c|c|c|c|c|c|c|}
\hline $\begin{array}{l}\text { Patient } \\
\text { No. }\end{array}$ & $\begin{array}{l}\text { Age, } \\
\text { years }\end{array}$ & \multicolumn{2}{|c|}{ Gender } & \multicolumn{3}{|c|}{ Type of tumor } & \multicolumn{3}{|c|}{$\begin{array}{l}\text { Tumor } \\
\text { grading }\end{array}$} & $\begin{array}{l}\text { Concurrent } \\
\text { chemotherapy }\end{array}$ & & \multicolumn{2}{|c|}{$\begin{array}{l}\text { Interval from RT to } \\
1^{\text {st }} \text { follow-up visit }\end{array}$} & $\begin{array}{l}\text { Interval from RT to } \\
\text { onset of symptoms }\end{array}$ \\
\hline 1 & 49.7 & \multirow{7}{*}{\multicolumn{2}{|c|}{$\begin{array}{l}\text { male } \\
\text { female } \\
\text { male } \\
\text { female } \\
\text { male } \\
\text { male } \\
\text { male }\end{array}$}} & \multicolumn{3}{|c|}{ G1 SCC } & \multicolumn{3}{|c|}{ T4a N0 M0 } & Yes & & \multicolumn{2}{|c|}{17.4 months } & 16.0 months \\
\hline 2 & 75.3 & & & \multicolumn{3}{|c|}{ G1 SCC } & \multicolumn{3}{|c|}{ T4a N0 M0 } & No & & \multicolumn{2}{|c|}{5.5 months } & 4.0 months \\
\hline 3 & 82.2 & & & \multicolumn{3}{|c|}{ G2/G3 SCC } & \multicolumn{3}{|c|}{ T4a N0 M0 } & No & & \multicolumn{2}{|c|}{10.0 months } & 4.0 months \\
\hline 4 & 71.1 & & & \multicolumn{3}{|c|}{ G3 undifferentiated } & \multicolumn{3}{|c|}{ T4a N0 M0 } & Yes & & \multicolumn{2}{|c|}{0.7 months } & -0.3 months \\
\hline 5 & 63.3 & & & \multicolumn{3}{|c|}{ G2 SCC } & \multicolumn{3}{|c|}{ T4b N2b M0 } & Yes & & \multicolumn{2}{|c|}{0.3 months } & -0.1 months \\
\hline 6 & 59.4 & & & \multicolumn{3}{|c|}{ G1/G2 SCC } & \multicolumn{3}{|c|}{ T4b N2c M0 } & Yes & & \multicolumn{2}{|c|}{2.8 months } & 1.5 months \\
\hline 7 & 60.7 & & & \multicolumn{3}{|c|}{ G1 SCC } & \multicolumn{3}{|c|}{ T4a N2b M0 } & Yes & & \multicolumn{2}{|c|}{0.6 months } & -0.1 months \\
\hline \multirow{2}{*}{$\begin{array}{l}\text { Patient } \\
\text { No. }\end{array}$} & \multicolumn{6}{|c|}{ Complications } & \multirow[b]{2}{*}{ RR } & \multirow[b]{2}{*}{$\mathrm{CP}$} & \multirow{2}{*}{$\begin{array}{l}\text { Initial } \\
\text { CDVA }\end{array}$} & \multirow{2}{*}{$\begin{array}{l}\text { Final } \\
\text { CDVA }\end{array}$} & \multicolumn{3}{|c|}{ Surgery } & Systemic outcome \\
\hline & LMD & DED & NK & LSCD & $\mathrm{CC}$ & glaucoma & & & & & TK & $\mathrm{AMT}$ & tarsorrhaphy & \\
\hline 1 & + & + & III & - & - & - & - & + & $6 / 12$ & PL & + & - & - & deceased \\
\hline 2 & + & + & III & - & + & - & - & + & $6 / 18$ & $\mathrm{HM}$ & - & + & + & disease-free \\
\hline 3 & + & + & I & + & + & + & + & - & $6 / 400$ & $\begin{array}{l}\text { PL (before } \\
\text { evisceration) }\end{array}$ & - & + & - & disease-free \\
\hline 4 & + & + & III & + & + & + & - & + & $6 / 18$ & $\mathrm{CF}$ & + & + & - & disease-free \\
\hline 5 & + & + & III & + & + & - & - & + & $6 / 36$ & $6 / 18$ & + & + & + & disease-free \\
\hline 6 & + & + & I & - & - & - & - & - & $6 / 9$ & $6 / 6$ & - & - & - & deceased \\
\hline 7 & + & + & II & - & + & - & - & - & $6 / 9$ & $6 / 9$ & - & + & + & recurrence \\
\hline
\end{tabular}

RT, radiotherapy; SCC, squamous cell carcinoma; CDVA, corrected-distance visual acuity; LMD, lid margin disease (including madarosis, meibomianitis, keratinization, and loss of meibomian glands); DED, dry eye disease; NK, neurotrophic keratopathy; LSCD, limbal stem cell deficiency; CC, cicatricial conjunctivitis; RR, radiation retinopathy; CP, corneal perforation; TK, tectonic keratoplasty; AMT, amniotic membrane transplant; G1, grade 1 (well-differentiated); G2, grade 2 (moderately differentiated); G3, grade 3 (poorly differentiated). ${ }^{1}$ The interval between the completion of radiotherapy and the first post-radiotherapy ophthalmic visit. ${ }^{2}$ The interval between the completion of radiotherapy and the onset of self-reported postradiotherapy symptoms. 

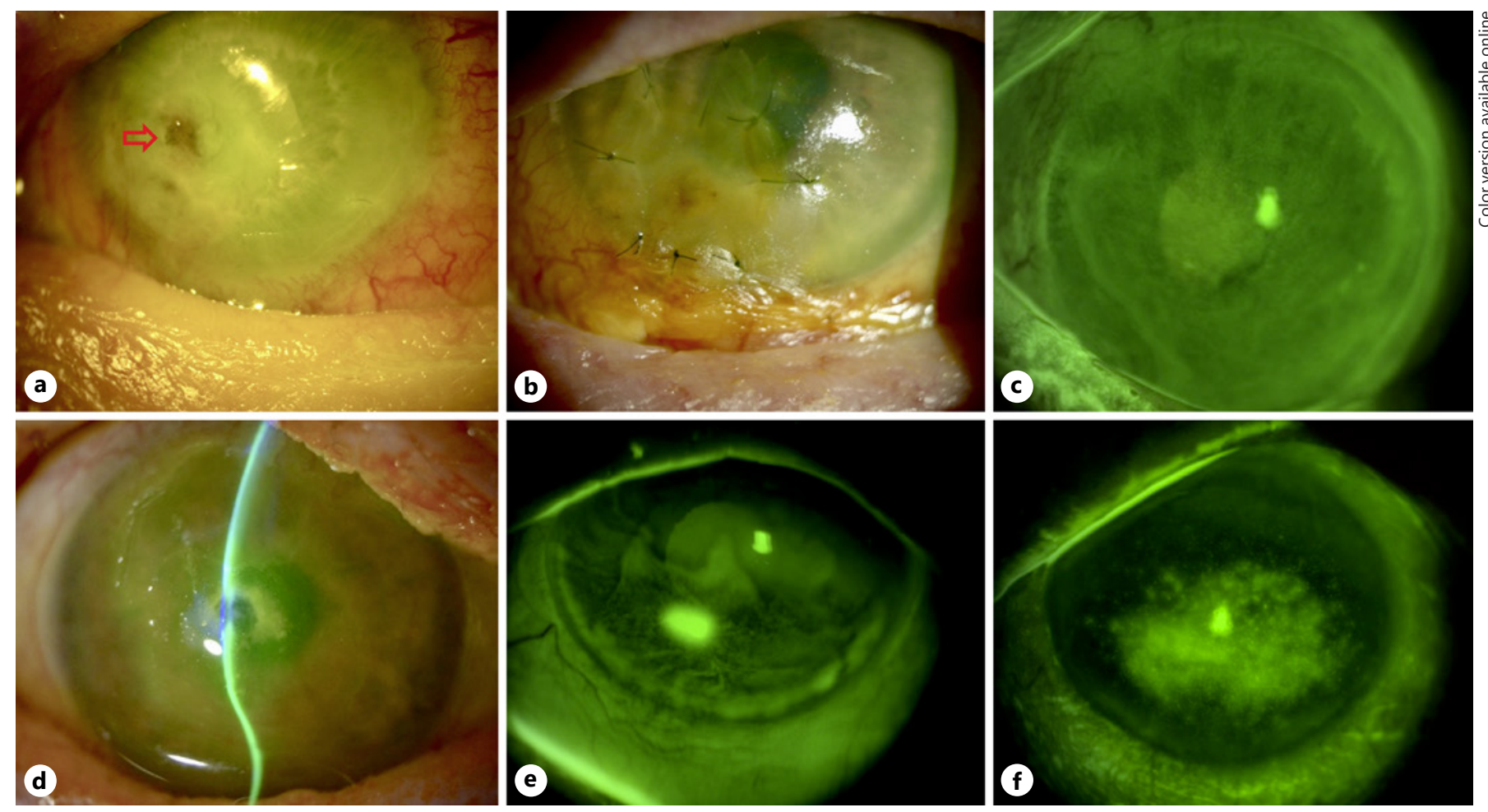

Fig. 2. An overview obtained by slit-lamp photography of the ocular and periocular complications following high-dose external beam radiotherapy for inoperable malignant maxillary sinus-involving tumors. a Patient 1: an area of corneal macro-perforation (red arrow) and complete madarosis. b Patient 2: a multilayered amniotic membrane transplant for descemetocoele. Note the complete madarosis and inferior conjunctival scarring and keratiniza-

\section{Ophthalmic Clinical Features}

The median intervals from the completion of EBRT to the onset of EBRT-related ocular symptoms was 1.5 months (interquartile range [IQR] -0.1 to 4 months); to the first post-EBRT ophthalmic visit, it was 2.8 months (IQR 0.6-10.0 months). During the study period, a wide range of periocular and ocular complications was noted (Fig. 2, 3). Severe meibomianitis, lid margin keratinization, partial-to-complete madarosis, dry eye disease (DED), reduced corneal sensation, and neurotrophic keratopathy (of varying grades) were universally observed in all patients (Table 1). Other complications included cicatricial conjunctivitis $(n=5,71 \%)$, corneal perforation $(n=4,57 \%)$, LSCD $(n=3,43 \%)$, symblepharon ( $n=3,43 \%)$, glaucoma $(n=2,29 \%)$, rubeosis secondary to radiation retinopathy $(n=1,14 \%)$, and superimposed candida keratitis $(n=1,14 \%)$.

Ocular Complications of Radiotherapy for Malignant MST tion. c Patient 3: widespread stippled fluorescein staining of the cornea, consistent with the diagnosis of limbal stem cell deficiency (LSCD). d Patient 4: grade 3 neurotrophic keratopathy with central corneal macro-perforation. e Patient 5: there is a small area of epithelial defect with diffuse stippled fluorescein staining of the inferior cornea, consistent with LSCD. f Patient 6: significant interpalpebral corneal staining.

\section{Ophthalmic Treatment and Outcomes}

All patients were treated with regular topical lubricants (during and after radiotherapy) and judicious use of topical steroids (only after the initial ophthalmic presentation). A range of ocular surgeries, including sutured and sutureless (PROKERA implant; Bio-Tissue, Miami, FL, USA) amniotic membrane transplant (AMT; 5, 71\%), tectonic keratoplasty $(3,43 \%)$, temporary/permanent tarsorrhaphy $(3,43 \%)$, transscleral cyclodiode laser (1, $14 \%)$, therapeutic corneal cross-linking or PACK-CXL $(1,14 \%)$ for refractory candida keratitis, and evisceration $(1,14 \%)$ were required (Table 1$)$. An evisceration was performed in Patient 3 to ameliorate the intractable ocular pain in view of the poor visual prognosis. The median corrected-distance visual acuity (CDVA) deteriorated from 20/60 (IQR 20/30-20/120) at the first postradiotherapy ophthalmic visit to counting fingers (range 20/ 30 - perception of light) at the final follow-up. Intact cor-

Ocul Oncol Pathol 2021;7:36-43 

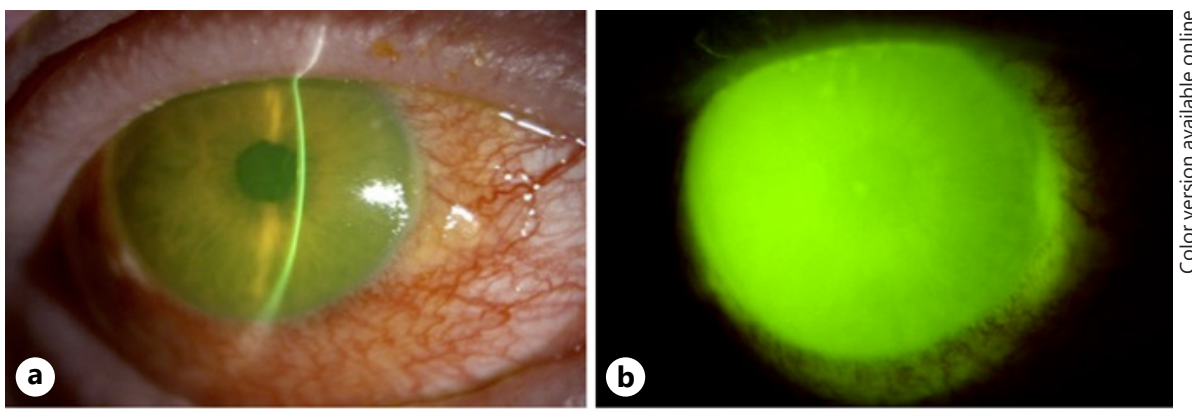

Fig. 3. Images obtained by slit-lamp photography of the right eye of Patient 7 . a, b Two weeks after radiotherapy, there was complete madarosis of the upper and lower eyelids, and severe conjunctival inflammation with complete corneal and perilimbal conjunctival epithelial defect. c, d Five weeks after radiotherapy, there was $60 \%$ epithelialization of the corneal and conjunctival epithelial defect superiorly after the insertion of a bandage contact lens device with amniotic membrane (PROKERA PLUS implant). e, f Three months after radiotherapy, there was a temporary tarsorrhaphy with complete epithelialization of the corneal and conjunctival defect.
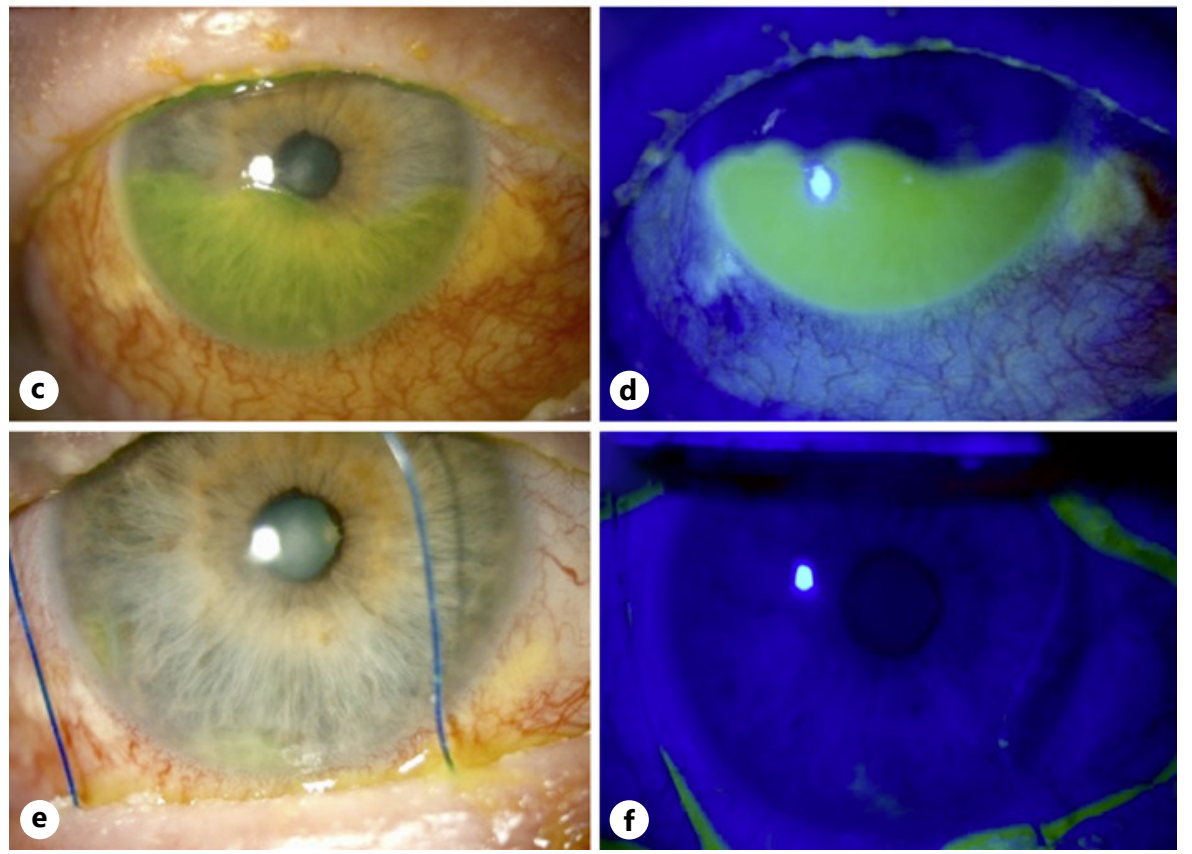

neal epithelium was observed in all patients (including Patient 3 before the evisceration) at final follow-up. Good visual outcome (defined as CDVA $\geq 20 / 60$ ) was achieved in $3(43 \%)$ patients, and complete remission of cancer was observed in 3 (43\%) patients at the final follow-up.

\section{Discussion}

In this case series, we highlight the clinical spectrum, management, and outcomes of ocular and periocular complications induced by high-dose EBRT for inoperable MMST with orbital involvement. We observed that all patients universally developed severe lid margin disease, ocular surface inflammation, DED, and neurotrophic keratopathy. Moreover, 4 (57\%) developed corneal perforation despite intensive medical and surgical treatment, and $1(14 \%)$ developed severe corneal neovascularization,
LSCD, and painful blinding neovascular glaucoma which necessitated an evisceration.

The toxic effect of radiotherapy on host tissues is primarily influenced by 3 factors, namely, the tolerance dose (TD) of host tissues to ionizing radiation, the radiotherapy dose per fraction, and the total cumulative radiation dose [9]. TD is frequently defined as either TD $5 / 5$ (a probability of a $5 \%$ complication rate at 5 years) or TD 50/5 (a probability of a 50\% complication rate at 5 years). Studies have shown that the TD $50 / 5$ is much lower for the ocular surface ( $50 \mathrm{~Gy}$ ) than for the retina (55 Gy) and optic nerve (>65 Gy) [9]. This explains the reason why ocular-surface complications are more commonly reported than retinopathy or optic neuropathy after ocular or periocular radiotherapy $[9,16,17]$. In addition, the risk of toxicity is dependent on the dose. Kwok et al. [17] reported that the incidence of severe radiation keratopathy increased from 0 to $100 \%$ when the radia-
40

Ocul Oncol Pathol 2021;7:36-43 DOI: $10.1159 / 000511011$
Ting et al. 
tion dose was increased from 59 to $>70$ Gy in patients who received radiotherapy for nasopharyngeal carcinoma. They also observed that lacrimal shielding reduced the risk of severe keratopathy [17]. Parsons et al. [18] similarly showed that a total radiation dose of $>57$ Gy for a range of orbital tumors was highly predictive of ocularsurface complications such as severe DED, corneal melt, and vascularization. These observations corroborate our findings where all the patients universally developed ocular-surface complications, including DED and neurotrophic keratopathy, after a total radiotherapy dose of 65 Gy in 30 fractions over 6 weeks using IMRT, the standard regime approved by the Royal College of Radiologists $[10,19]$.

Interestingly, Graue et al. [20] observed a considerably lower rate of ocular-surface complications despite a high dose of radiation (50-60 Gy) for conjunctival/corneal SCC. This lower rate of complications was related to the size and superficial location of the tumors, which means that EBRT or superficial X-ray therapy (SXT) can be used. In contrast, deep-seated orbital tumors require high-energy photons to be delivered externally from many different angles to achieve full and conformal radiation of the entire tumor. The high rate of complications observed in our study might also have been related to treatment delay. Although all our patients were treated with topical steroids (clinically indicated), it was unlikely that the infrequent low dose contributed to the high rate of complications. Lacrimal shielding was not deployed in our patients because it can only be used in orthogonal-ray treatments (when a radiation beam is delivered at $90^{\circ}$ to the tumor) such as in SXT or electron treatments for superficial tumors. With IMRT, such shielding would interfere with radiation delivery and increase the risk of treatment failure. Instead, we employed a more sophisticated technique, IMRT with unique multileaf collimators that shape multiple beamlets, providing shielding within the treatment head to conform to the tumor rather than placing the shielding on the patients [10].

Modern linear accelerators delivering IMRT have the capability of providing a daily CT image. This is principally used to detect small positional and anatomical changes, day to day, so that minor adjustments to the position of the patient can be made. This is known as image-guided radiotherapy (IGRT). It can prevent geometric misses and improve outcomes. IGRT can also detect weight loss and internal organ movement (e.g., in the pelvis). These kinds of adjustments to a radiotherapy treatment plan are known as "adaptive radiotherapy"

Ocular Complications of Radiotherapy for Malignant MST
[21]. One could theoretically change a radiotherapy plan for shrinkage of a tumor, but several problems are associated with this. First, on-treatment images are not currently of sufficiently high quality to recognize a change in tumor volume in an area of soft tissue like the orbit. Second, the planning process is very time-consuming and often would not allow the timely production of a new plan, particularly with SCC, which is a "category 1" tumor, meaning that delays during mid-treatment can result in accelerated repopulation of tumor cells and a worse outcome. Third, adjusting the clinical target volume (CTV) during treatment for a shrinking tumor would potentially miss microscopic disease, and not conform to the International Commission on Radiation Units (ICRU) published recommendations [22]. However, replanning is carried out for external contour changes, which can be seen with daily imaging. In our case series, this was carried out for Patient 6, not because the tumor had shrunk significantly during treatment, but because the patient had lost weight due to nausea, mucositis, and soreness on swallowing. SCCs will shrink during treatment, but they are not the most radiosensitive of tumors and the process is gradual, making any adaptive replanning being of only limited theoretical benefit. The best intervention to avoid radiation toxicity to the eye in MMSTs invading the orbit would be early detection, at a stage when the tumor is smaller and away from the eye, making surgical resection an option, or making the radiotherapy volume smaller.

Depending on the radiotherapy dose and treatment regime, the onset of radiotherapy-related ocular symptoms and signs can range from weeks, months to years $[9,10]$. However, in patients who have received high-dose radiotherapy (e.g., >57 Gy), corneal opacification and vascularization usually become evident by $9-10$ months [18] In our series, the median interval between EBRT and the manifestation of EBRT-related ocular symptoms/signs was 1.5 months (IQR -0.1.0 to 4 months). In fact, 3 patients started developing some level of ocular discomfort towards the end of the 6-week EBRT. During the initial phase of our study, the routine practice was that patients who received high-dose EBRT for MMST were only referred to the ophthalmology team if they complained of considerable ocular symptoms (a reactive approach). This was reflected by the long interval between the completion of EBRT and the first post-EBRT ophthalmic visit (ranging from 5.5 to 16 months) in the first 3 patients (Patients 1-3). The delay in referral might be partly related to the underlying disease severity itself, and partly attributed to the underlying corneal hypoesthesia ob-

Ocul Oncol Pathol 2021;7:36-43

DOI: 10.1159/000511011 
served in all patients, causing the patients and the nonophthalmic health professionals including the oncologists, radiologists, and maxillofacial surgeons to underestimate the severity of the ocular disease.

During the later stage of our study, the patients were referred to the ophthalmologists, regardless of whether they were symptomatic or not, for regular monitoring for any evolving ophthalmic complication after the EBRT (a proactive approach), which enabled timely ophthalmic interventions. This was reflected by the good final visual outcome observed in 3 patients (Patients 5-7). An in vitro murine model of radiation keratopathy recently reported by Harris et al. [23] demonstrated significant loss of corneal nerves with the influx and activation of immune cells within months of sublethal radiation. Unfortunately, no in vivo confocal microscopy facility was available in our unit to visualize the extent of damage to the corneal nerve. In addition, systemic chemotherapy is known to cause ocular side effects, but these are rarely observed with the use of cisplatin which can result in posterior segment toxicity (if it does occur) [24].

In our experience, AMT serves as a potentially useful treatment modality to improve and restore the ocular surface integrity in severe radiation keratopathy. All patients had maintained an intact corneal epithelium at the final follow-up. A notable example was observed in Patient 7, who presented with complete corneal and perilimbal conjunctival epithelial defect at 2 weeks post-EBRT. He achieved rapid corneal healing within weeks via sutureless AMT (PROKERA implant), followed by temporary tarsorrhaphy (Fig. 3). AMT promotes corneal epithelialization and exhibits anti-inflammatory, antifibrotic, antiangiogenic, and antimicrobial properties [25]. It has been used to treat persistent epithelial defects, chemical eye injury, Steven-Johnson syndrome, infectious keratitis, corneal perforation, bullous keratopathy, LSCD, conjunctival reconstruction, and DED [25-28]. Future studies evaluating the prophylactic role of AMT in reducing the risk and severity of radiation keratopathy after highdose orbital/periorbital EBRT will be valuable. In view of the potential utility of PACK-CXL in treating fungal keratitis, $[29,30]$ this adjuvant treatment was employed to treat 1 patient affected by refractory candida keratitis (Patient 4). However, the cornea failed to respond to the treatment and perforated 2 days later, which necessitated an emergency tectonic keratoplasty.

It is interesting to note that our patients had varying outcomes despite having received the same radiotherapy treatment regime (although not all received concurrent chemotherapy, which can increase radiation-related toxicities). First, this might be partly related to the delayed ophthalmic review in the first 3 patients (final CDVA of HM-evisceration) compared to the last 3 patients (final CDVA of 20/20-20/60). Second, this was influenced by the location where the maximal dose of radiation was administered in relation to the globe and other critical optical structures (e.g., how close the high dose was to the globe). If the high-dose treatment volume was away from the globe, the patients were less likely to develop ocularsurface complications and vice versa. Therefore, to some extent, it is possible for the oncologist to predict the severity of the periocular and ocular diseases following radiotherapy, highlighting the importance of good multidisciplinary teamwork between ophthalmologists and oncologists to optimize patient care. However, there is also an unpredictable element, which is the variation in normaltissue sensitivity to radiation between patients, and this can be different for both early and late side effects. These are known as "host factors" and include genetic and lifestyle factors [31].

In conclusion, we have highlighted the clinical spectrum and challenges in managing the periocular and ocular complications associated with high-dose radiotherapy for inoperable MMST. AMT serves as a potentially useful therapeutic modality in restoring the ocular surface integrity following high-dose radiotherapy. Good multidisciplinary teamwork by ophthalmologists, maxillofacial surgeons, clinical oncologists, and radiologists is essential for optimizing the outcome of this challenging clinical entity and maximizing the chance of preserving the eye. In view of the high risk of complications, we advocate a proactive approach in monitoring for any evolving ophthalmic complications during and after the EBRT.

\section{Statement of Ethics}

Ethics approval was not required as this retrospective study was classified as a clinical service evaluation. All treatment provided was part of the standard clinical care and the conduct of study adhered to the tenets of Declaration of Helsinki. Written consent was obtained from all patients for the procedures and surgeries described in this study and for publication.

\section{Conflict of Interest Statement}

The authors have no conflicts of interest to declare.
Ocul Oncol Pathol 2021;7:36-43 DOI: $10.1159 / 000511011$
Ting et al. 


\section{Funding Sources}

D.S.J.T. acknowledges support by the Medical Research Council/Fight for Sight Clinical Research PhD Fellowship (MR/ T001674/1), and the Fight for Sight/John Lee, Royal College of Ophthalmologists Primer Fellowship (24CO4).

\section{Author Contributions}

D.S.J.T. and T.P. conceptualization and design of study. R.R.R., J.Y.N., and D.J.P.W. data collection. D.S.J.T., D.J.P.W., D.A.-K., and T.P. data analysis and interpretation. D.S.J.T. manuscript drafting. R.R.-R., J.Y.N., D.J.P.W., D.A.-K., and T.P. critical review of manuscript. All authors approved the final version.

\section{References}

1 Ansa B, Goodman M, Ward K, Kono SA, Owonikoko TK, Higgins K, et al. Paranasal sinus squamous cell carcinoma incidence and survival based on Surveillance, Epidemiology, and End Results data, 1973 to 2009. Cancer. 2013 Jul;119(14):2602-10.

2 Lund VJ, Clarke PM, Swift AC, McGarry GW, Kerawala C, Carnell D. Nose and paranasal sinus tumours: United Kingdom National Multidisciplinary Guidelines. J Laryngol Otol. 2016 May;130(S2):S111-8.

3 McCary WS, Levine PA. Management of the eye in the treatment of sinonasal cancers. Otolaryngol Clin North Am. 1995 Dec;28(6): 1231-8.

4 Barrett A, Dobbs J, Morris S, Roques T. Practical Radiotherapy Planning. 4th ed. CRC Press; 2009. p. 186.

5 Baskar R, Dai J, Wenlong N, Yeo R, Yeoh KW. Biological response of cancer cells to radiation treatment. Front Mol Biosci. 2014 Nov;1: 24.

6 Steel GG, McMillan TJ, Peacock JH. The 5Rs of radiobiology. Int J Radiat Biol. 1989 Dec; 56(6):1045-8.

7 Baskar R, Lee KA, Yeo R, Yeoh KW. Cancer and radiation therapy: current advances and future directions. Int J Med Sci. 2012;9(3): 193-9.

8 Bortfeld T. IMRT: a review and preview. Phys Med Biol. 2006 Jul;51(13):R363-79.

9 Durkin SR, Roos D, Higgs B, Casson RJ, Selva D. Ophthalmic and adnexal complications of radiotherapy. Acta Ophthalmol Scand. 2007 May;85(3):240-50.

10 Finger PT. Radiation therapy for orbital tumors: concepts, current use, and ophthalmic radiation side effects. Surv Ophthalmol. 2009 Sep-Oct;54(5):545-68.

11 Tiwari S, Bhatt A, Nagamodi J, Ali MJ, Ali H, Naik MN, et al. Aqueous Deficient Dry Eye Syndrome Post Orbital Radiotherapy: A 10Year Retrospective Study. Transl Vis Sci Technol. 2017 Jun;6(3):19.
12 Fujishima H, Shimazaki J, Tsubota K. Temporary corneal stem cell dysfunction after radiation therapy. Br J Ophthalmol. 1996 Oct; 80(10):911-4

13 Amin MB, Greene FL, Edge SB, et al. The Eighth Edition AJCC Cancer Staging Manual: Continuing to build a bridge from a population-based to a more "personalized" approach to cancer staging. CA Cancer J Clin. 2017;67: 93-9.

14 Mackie IA. Neuroparalytic keratitis. In: Fraunfelder FT, Roy FH, Grove J, editors. Current Ocular Therapy. 4th ed. Philadelphia: W. B. Saunder; 1995. pp. 452-4.

15 Deng SX, Borderie V, Chan CC, Dana R, Figueiredo FC, Gomes JA, et al.; and The International Limbal Stem Cell Deficiency Working Group. Global consensus on definition, classification, diagnosis, and staging of limbal stem cell deficiency. Cornea. 2019 Mar; 38(3):364-75.

16 Gore SK, Plowman NP, Dharmasena A, Verity DH, Rose GE. Corneal complications after orbital radiotherapy for primary epithelial malignancies of the lacrimal gland. $\mathrm{Br} \mathrm{J} \mathrm{Oph}-$ thalmol. 2018 Jul;102(7):882-4.

17 Kwok SK, Ho PC, Leung SF, Gandhi S, Lee VW, Lam DS, et al. An analysis of the incidence and risk factors of developing severe keratopathy in eyes after megavoltage external beam irradiation. Ophthalmology. 1998 Nov;105(11):2051-5.

18 Parsons JT, Bova FJ, Fitzgerald CR, Mendenhall WM, Million RR. Severe dry-eye syndrome following external beam irradiation. Int J Radiat Oncol Biol Phys. 1994 Nov;30(4): 775-80.

19 The Royal College of Radiologist. Chapter 6: Head and neck cancer. In: Clinical Oncology: Radiotherapy dose fractionation, 3rd edition 2019; pp. 41-9.

20 Graue GF, Tena LB, Finger PT. Electron beam radiation for conjunctival squamous carcinoma. Ophthal Plast Reconstr Surg. 2011 JulAug;27(4):277-81.

21 Sibtain A, Morgan A, MacDougall N. Radiotherapy inn practice: physics for clinical oncology. New York, USA: Oxford University Press; 2012.
22 International Commission on Radiation Units and Measurements. Prescribing, recording and reporting photon beam therapy. Report 50. Bethesda, Maryland, USA: ICRU; 1993.

23 Harris DL, Yamaguchi T, Hamrah P. A novel murine model of radiation keratopathy. Invest Ophthalmol Vis Sci. 2018 Aug;59(10): 3889-96.

24 Omoti AE, Omoti CE. Ocular toxicity of systemic anticancer chemotherapy. Pharm Pract (Granada). 2006 Apr;4(2):55-9.

25 Jirsova K, Jones GL. Amniotic membrane in ophthalmology: properties, preparation, storage and indications for grafting-a review. Cell Tissue Bank. 2017 Jun;18(2):193-204.

26 Ting DS, Henein C, Said DG, Dua HS. Effectiveness and safety of early adjuvant amniotic membrane transplant versus standard antimicrobial therapy for infectious keratitis: a systematic review protocol. JBI Evid Synth. 2020 Aug; 18(8):1808-14.

27 Dua HS, Ting DS, Al Saadi A, Said DG. Chemical eye injury: pathophysiology, assessment and management. Eye (Lond). 2020 Jun; DOI: 10.1038/s41433-020-1026-6.

28 Sharma N, Thenarasun SA, Kaur M, Pushker $\mathrm{N}$, Khanna N, Agarwal T, et al. Adjuvant role of amniotic membrane transplantation in acute ocular Stevens-Johnson syndrome: A randomized control trial. Ophthalmology. 2016 Mar;123(3):484-91.

29 Ting DS, Henein C, Said DG, Dua HS. Photoactivated chromophore for infectious keratitis - Corneal cross-linking (PACK-CXL): A systematic review and meta-analysis. Ocul Surf. 2019 Oct;17(4):624-34.

30 Ting DS, Mckenna M, Sadiq SN, Martin J, Mudhar HS, Meeney A, et al. Arthrographis kalrae keratitis complicated by endophthalmitis: A case report with literature review. Eye Contact Lens. 2020 May; DOI: 10.1097/ ICL.0000000000000713.

31 Marks LB, Yorke ED, Jackson A, Ten Haken RK, Constine LS, Eisbruch A, et al. Use of normal tissue complication probability models in the clinic. Int J Radiat Oncol Biol Phys. 2010 Mar;76(3 Suppl):S10-9. 